\title{
Clinical features of vertical sight disorders in patients with parkinson's disease
}

\section{Khaydarova Dildora Kadirovna ${ }^{1}$, Khodjieva Dilbar Tadjiyevna ${ }^{1}$}

${ }^{1}$ Department of Neurology and psychiatry, Bukhara Medical Institute, Republic of Uzbekistan

\section{Khaydarov Nodirjon Kadirovich ${ }^{2}$}

${ }^{2}$ Tashkent Institute of Advanced Medical Education, Republic of Uzbekistan

\section{Email address:}

dildora_doktor@mail.ru (Khaydarova Dildora Kadirovna)

\section{To cite this article:}

Khaydarova Dildora Kadirovna, Khodjieva Dilbar Tadjiyevna, Khaydarov Nodirjon Kadirovich. Clinical features of vertical sight disorders in patients with parkinson's disease. Journal of research in health science. Vol. 1, No. 2, 2018, pp. 14-17. DOI 10.26739/2523-1243

\section{d ol ${ }^{\circ}$ http://dx.doi.org/10.26739/2523-1243/-2018-1-2-2}

\begin{abstract}
Insufficiency of the basal ganglia in Parkinson's disease (PD) leads to characteristic motor symptoms in the limbs, axial musculature. A correct evaluation of the oculomotor function in patients with PDshould be conducted taking into account the interaction of the visual and vestibular systems. When studying vertical oculomotor reflexes in patients with PD, close attention is required to study the state of the functional system of the "eyelid", which adapts the movements of the eyelids to the movements of the eyeball. The distinctive features of the nystagmic cycles themselves in patients with PD caused by optoclinic stimulation, namely the change in the ratio of the duration of the fast and slow phases of the nystagmus, are revealed.
\end{abstract}

Keywords: nystagmic cycles, Parkinson's disease, optokineticnystagmus, vestibulo-ocular reflexes.

\section{Introduction}

Human movements are complex phenomenarealized with the participation of pyramidal, extrapyramidal and cerebellar systems, whose work is largely determined by the safety of sensory afferentation and the state of higher formations that perform coordination between sensory and motor processes.
Insufficiency of the basal ganglia in Parkinson's disease (PD) leads to characteristic motor symptoms in the limbs, axial musculature. The oculomotor system as part of the general motor function is also involved in pathological changes caused by PD, and if at the expressed stages of the disease it is not uncommon to see vertical vision 
restriction in a routine neurological examination, then in the initial stages such disorders can be detected only with the use of special equipment.

A correct evaluation of the oculomotor function in patients with PDshould be conducted taking into account the interaction of the visual and vestibular systems. It is known that the basal ganglia control the brain stem structures that generate fast and slow phases of the nystagmus, as well as other nonvestibular types of eye movements that are disrupted in neurodegenerative diseases. Close cooperation with the visual system ensures the coordinated work of all links of the oculomotor apparatus, which is important for the implementation of friendly eye movements and merging into a single whole of static and dynamic objects. This is achieved with the help of the following complex reflexes: tracking, optokineticnystagmus (OKN), vestibuloocular reflexes (VOR), saccades, etc. In addition, the "visual-oculomotor" system can be considered as a model for studying the interaction between sensory and motor processes in PD. In PD, the study of vertical oculomotor reflexes is of great importance, since they are in close conjugation with the gravitational system, the change in the state of which makes a considerable contribution to the pathophysiological mechanisms of postural instability, a violation of maintaining the vertical posture and walking. When studying vertical oculomotor reflexes in patients with PD, close attention is required to study the state of the functional system of the "eyelid", which adapts the movements of the eyelids to the movements of the eyeball.
Oculomotor disorders in PDcan be conditionally divided into quantitative and qualitative ones.

\section{The quantitative include:}

- Reducing the speed of arbitrary and reflex saccades;

- Decrease in speed and smoothness of follow-up movements of eyes after a smoothly moving object;

- Paresis of arbitrary vertical gaze (at the advanced stages of the disease);

- Rare flashing;

- "frozen" look.

Qualitative oculomotor disorders

\section{include:}

- Choreic hyperkinesia of eyeballs;

- Tremor of eyeballs and eyelids;

- Ptosis;

- Apraxia of the eyelid rises;

- Blepharospasm.

The aim of the study was to study vertical oculomotor reflexes and the state of the functional "eyelid" system in patients with PD.

Eye and eyelid movements were evaluated during clinical examination, as well as during video-histogramography on the "Interacoustcs" hardware complex (Denmark). 285 patients with PD at the age of 30 to 70 years were examined.

It has been found that with PD, the speed response of oculomotor reactions in all the tests used is slowing, the latent time increases and the accuracy of the saccades decreases when performing the saccadic test. The rate of smooth tracking and the speed of the slow phase of the OKN decrease, and these vertical disturbances manifest themselves in greater degree than in the horizontal. There is a direct correlation between the severity of oculomotor dysfunction and the severity of the disease, irrespective of its duration. 
Khaydarova Dildora Kadirovna1, Khodjieva Dilbar Tadjiyevna, Khaydarov Nodirjon Kadirovich. Clinical features of vertical sight disorders in patients with parkinson's disease.

When sinusoidal rotation is performed around the axial axis in the dark, a vertical rotational nystagmus is recorded, the intensity of which can also be variable and depends on the modulating influence of higher cerebral structures on the arc of the vertical VOR. The test is evaluated in terms of the speed of the slow phase of the nystagmus (SSP) up and down. Investigation of the suppression of vertical VOR by fixing the gaze on an object moving with the head, gives useful information about the state of visualvestibular interaction, reflecting the functioning of brain stem structures. A characteristic feature of all PD patients is a violation of suppression of vertical nystagmus in both directions by fixing the gaze on a moving object with the head already in the early stages of the disease. With the increase in the stage of the disease, the suppression of nystagmus by fixation worsens even more, and in patients with stage 3-4 of the PD, the values of the CMF of the vertical nystagmus up and/or down often exceed the angular velocity of the head.

The distinctive features of the nystagmic cycles themselves in patients with PD caused by optoclinic stimulation, namely the change in the ratio of the duration of the fast and slow phases of the nystagmus, are revealed. Normally, this ratio of SBP/SSP is $1: 8$, with PD 1: 3-4.

The study of the function of the upper eyelid began to attach great importance only in recent times. Neurophysiological registration, analysis and quantitative determination of the severity of this or that motor disorder on the part of the century are currently not sufficiently developed or lacked in general due to the lack of proper equipment and / or software and hardware systems.

The problem of registering eye movements was solved using the videooculography method, and to simplify the registration of movements of the upper eyelid, a paper marker of a circular shape was fixed on it. The image of the eye and the upper eyelid with the marker is made with the help of an infrared camera. Each frame of the received video is processed on the computer with the help of the generalized Hafa transformation algorithm in the authors' modification in such a way that the coordinates of the center of the pupil and the marker are measured on each frame during the test.

Loss of coordination in the system "eyelid-eye" is best manifested in the presentation vertical optokinetic stimulation, the smooth tracking test or provocation vertical vestibular-ocular reflex. It has been found that the most complete information about the violation coordinated operation century and eyes can be obtained by analyzing the correlation coefficients (Pearson, Spearman) between the moving speeds of the pupil and the time tags, and in measuring the phase shift between the movements of the eyes and age.

It was found that in healthy subjects achieved zero shear maximum correlation. Any shift of zero indicates a violation of coordinated movements of the century and the eyes.

When examining patients, we identified the following main patterns of disorders in the eyelid system:

- Lag of eyelid movements from the eye (mainly in patients with progressive supranuclear palsy);

- Eyelids move ahead of the eye (in a number of patients with severe PD stages); 
- Eyelid and eye move in antiphase (in patients with pronounced choreic hyperkinesia);

- Nystagmus of the eyelids with optokinetic stimulation in patients with severe stages of the disease, in which significant vertical disturbances were observed in the absence of vertical OKN.

Thus, in patients with $\mathrm{PD}$, a violation in the vertical vision system is one of the symptoms of this disease. Registration and quantitative analysis of the parameters of the main oculomotor reflexes will allow to assess the adequacy of the prescribed therapy, as well as to solve some expert questions, and the proposed method of quantitative assessment of disorders in the eyelid system opens new diagnostic perspectives in studying the pathology of the vertical gaze. 